\title{
Kidney- and Site-Selective Delivery of 5-Fluorouracil Utilizing the Absorption on the Kidney Surface in Rats
}

\author{
Shigeru KawaKami, ${ }^{*}, a$ Tomomi Horimoto, ${ }^{a}$ Koyo Nishida, ${ }^{a}$ Ryu Hirayama, ${ }^{a}$ Takahiro MukaI, ${ }^{a}$ \\ Mikiro NaKashima, ${ }^{b}$ Hitoshi SASaki, ${ }^{b}$ Toshiyuki SaKaedA, ${ }^{c}$ and Junzo NaKamura ${ }^{a}$ \\ ${ }^{a}$ School of Pharmaceutical Sciences, Nagasaki University; 1-14 Bunkyo-machi, Nagasaki 852-8521, Japan: ${ }^{b}$ Department \\ of Hospital Pharmacy, Nagasaki University School of Medicine; 1-7-1 Sakamoto, Nagasaki 852-8501, Japan: and \\ ${ }^{c}$ Department of Hospital Pharmacy, School of Medicine, Kobe University; 7-5-2 Kusunoki-cho, Chuo-ku, Kobe 650-0017, \\ Japan. Received February 25, 2002; accepted April 9, 2002
}

The present study was undertaken to elucidate the kidney- and site-selective delivery of 5-fluorouracil (5FU) utilizing the absorption on the kidney surface in rats. An experimental system utilizing a cylindrical diffusion cell attached to the right kidney surface was established. After intravenous administration of 5-FU, the concentration of 5-FU in the right and left kidneys was almost the same and was rapidly eliminated. After right kidney surface application of 5-FU, however, the concentration of 5-FU in the right kidney was significantly higher than in the left kidney and other tissues. The 5-FU concentration in four sites of the right kidney after intravenous administration was almost the same. In contrast, 5-FU was site selectively delivered in the kidney after kidney surface application. The blood concentration of 5-FU was low $(<1.7 \mu \mathrm{g} / \mathrm{ml})$ until 120 min after kidney surface application. The maximum blood concentration of 5-FU after kidney surface application was much lower than after intravenous administration.

Key words 5 -fluorouracil; kidney; targeting; drug delivery system; rat; anticancer drug

Renal cell carcinoma is the common name applied to adenocarcinoma of the kidney, a tumor arising in the kidney. In recent years, the tendency to perform ultrasonography, computed tomography, or even magnetic resonance imaging has led to increased detection of small, localized, and asymptomatic tumors. ${ }^{1)}$ The current principal treatment for renal cell carcinoma is surgical nephrectomy. For the majority of patients who have metastatic or advanced renal cell carcinoma that is not curable surgically, chemotherapy remains of limited value because response rates of less than $20 \%$ are achieved. ${ }^{2}$ Therefore kidney- and site-selective delivery methods must be developed for anticancer drugs for the clinical treatment of renal cell carcinoma. To enhance the efficacy of anticancer drugs and to reduce their toxicity, much attention has been focused on renal cell carcinoma. The renal arterial administration of poly (stylene-co-maleic acid)-conjugated neocarzinostatin (SMANCS)/lipiodol, ${ }^{3,4)}$ microcapsule chemoembolization, ${ }^{5,6)}$ or doxorubicin-incorporated microspheres $^{7,8)}$ were studied to target delivery of anticancer drugs to the unilateral kidney. The combined uses of various anticancer drugs by intravenous and oral administration were reported to enhance the therapeutic effects against renal cell carcinoma. ${ }^{9)}$ When the drugs were administered by the vasculature route, they were distributed through the whole body via the blood stream, leading to inadequate kidney- and siteselective drug delivery.

In the present study, we examined the kidney- and site-selective delivery of 5-fluorouracil (5-FU) utilizing absorption on the kidney surface in rats.

\section{MATERIALS AND METHODS}

Chemicals 5-FU was purchased from Nacalai Tesque, Inc. (Kyoto, Japan). All other chemicals were of reagent grade.

In Vivo Experiments All experiments in the present study were carried out in accordance with the Guidelines for
Animal Experimentation of Nagasaki University. Male Wistar rats $(260-310 \mathrm{~g})$ were anesthetized with sodium pentobarbital $(50 \mathrm{mg} / \mathrm{kg}$ i.p.). After the right peritoneum was dissected about $3 \mathrm{~cm}$, a cylindrical diffusion cell (i.d. $9 \mathrm{~mm}$, effective area $0.64 \mathrm{~cm}^{2}$ ) was attached to the right kidney surface with Aron Alpha (Sankyo Co., Ltd., Tokyo, Japan), and 5 -FU $(50 \mathrm{mg} / \mathrm{ml} \times 0.1 \mathrm{ml})$ was added to the diffusion cell directly (Chart 1 ). The body temperature of the rats was maintained at $37^{\circ} \mathrm{C}$ with a heat lamp during the experiment. As control experiments, 5 -FU $(50 \mathrm{mg} / \mathrm{ml} \times 0.1 \mathrm{ml})$ was injected into the jugular vein. At the predetermined times, the rats were killed, and the liver, lung, right kidney, left kidney, spleen, heart, and lung were removed. To evaluate the intrarenal distribution of 5-FU, the kidney was divided from the reniportal structure, and they were further separated into the site under the diffusion cell and the site not under the diffusion cell (Chart 2). The tissues were weighed and homogenized in a two-fold volume of phosphate-buffered saline, $\mathrm{pH}$ 7.4 .

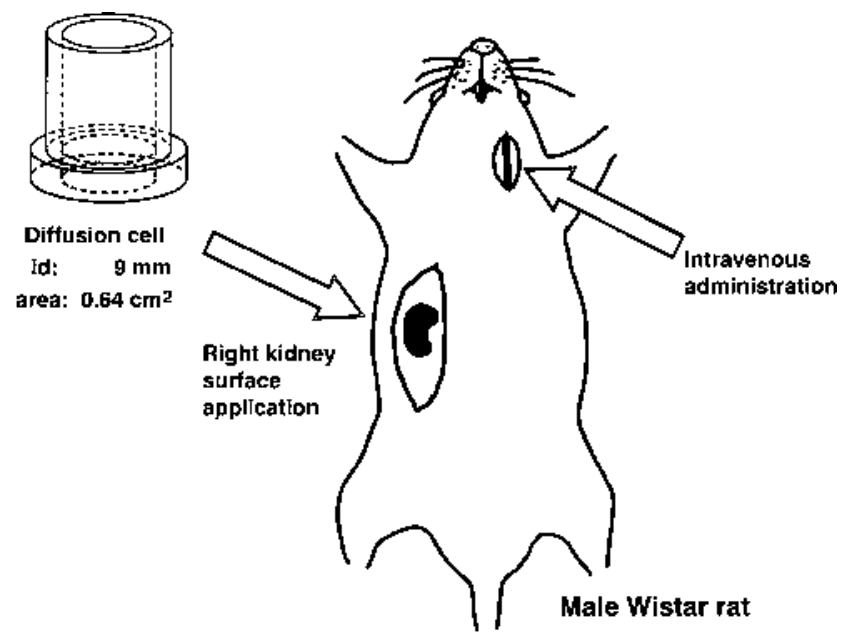

Chart 1. Experimental Procedures 


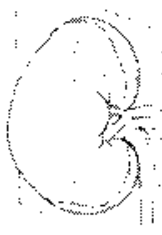

Applied kidney

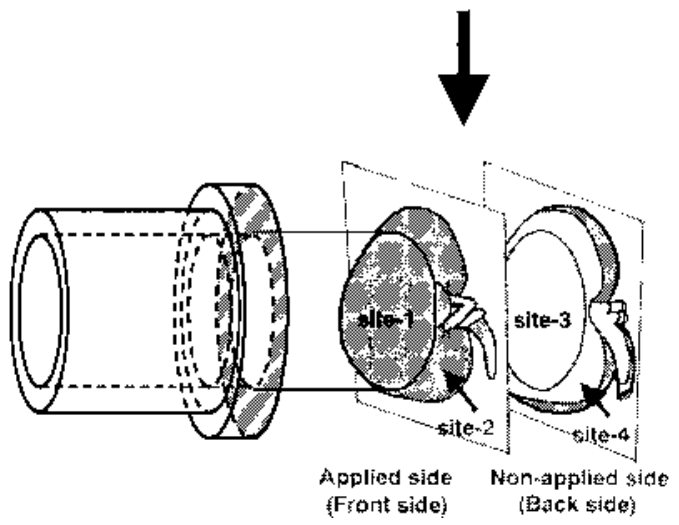

Chart 2. Division of Kidney

5-FU Assay Procedures in Blood and Tissues The concentration of 5-FU in each tissue homogenate or blood was determined by modifying the reported methods. ${ }^{10,11)}$ Briefly, the tissue homogenates $(300 \mu \mathrm{l})$ and blood samples $(300 \mu \mathrm{l})$ were added to a solution of 5-bromouracil $(20 \mu \mathrm{g} / \mathrm{ml}, 150 \mu \mathrm{l})$ dissolved in isotonic phosphate buffer ( $\mathrm{pH}$ 7.4) as an internal standard, $1 \mathrm{~m}$ sodium acetate buffer $(\mathrm{pH} 4.8,100 \mu \mathrm{l})$, and $20 \%$ anhydrous sodium sulfate $(500 \mu \mathrm{l})$. The mixtures were shaken with ethyl acetate $(4 \mathrm{ml})$ for $10 \mathrm{~min}$ and centrifuged at $900 \times \boldsymbol{g}$ for $10 \mathrm{~min}$. The organic layers $(3 \mathrm{ml})$ were collected. Then ethyl acetate $(4 \mathrm{ml})$ was added to the residue and the mixtures were shaken for $10 \mathrm{~min}$, and thereafter centrifuged at $900 \times \boldsymbol{g}$ for $10 \mathrm{~min}$. The organic layers $(4 \mathrm{ml})$ were collected and the mixed organic layers $(7 \mathrm{ml})$ were evaporated. The extraction residues were dissolved in $500 \mu \mathrm{l}$ of distilled water and were washed twice with $1.0 \mathrm{ml}$ of hexane. Samples $(100 \mu \mathrm{l})$ were injected onto the HPLC column. The recovery rates for extraction of 5-FU from blood and each tissue homogenate were approximately $75 \%$. The detection limits of 5 -FU in the blood and each tissue homogenate were $0.2 \mu \mathrm{g} /$ ml. An HPLC system (LC-6A, Shimadzu Co., Ltd., Kyoto, Japan) with a variable-wavelength UV detector (SPD-10A, Shimadzu) was used in reverse-phase mode. The detector wavelength, flow rate, and column temperature were set at $266 \mathrm{~nm}, 0.7 \mathrm{ml} / \mathrm{min}$, and $25^{\circ} \mathrm{C}$, respectively. The mobile phase consisted of $10 \mathrm{~mm}$ sodium acetate buffer ( $\mathrm{pH} 4.0$ ). The stationary phase used was a YMC-Pack ODS-A A-302 column $(150 \mathrm{~mm}$ length $\times 4.6 \mathrm{~mm}$ i.d., YMC Inc., Kyoto, Japan).

Statistical Analysis Statistical comparisons were performed using analysis of variance. $p<0.05$ was considered to be indicative of statistical significance.

\section{RESULTS AND DISCUSSION}

5-FU is commonly used in clinical oncology practice and

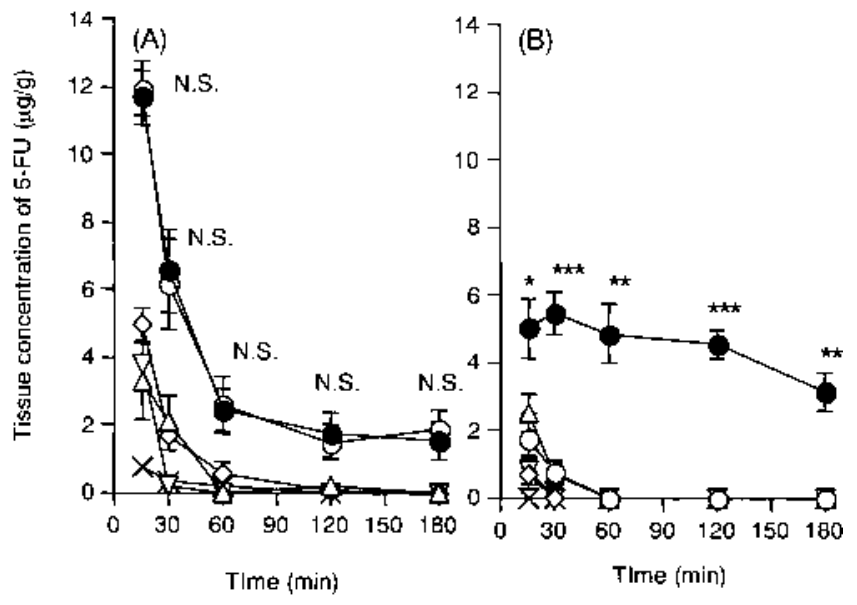

Fig. 1. Tissue Concentration of 5-FU after Intravenous Administration (A) or Kidney Surface Application (B) of 5-FU to Rats at a Dose of $5 \mathrm{mg}$

5-FU was determined in the right kidney $(\bullet)$, left kidney $(\bigcirc)$, liver $(\triangle)$, spleen $(\diamond)$, heart $(\nabla)$, and lung $(\times)$. Statistical comparisons were performed using analysis of variance $(* p<0.05, * * p<0.01, * * * p<0.0001$, significantly different from the left kidney; N.S., not significant). Each value represents the mean \pm S.E. of at least four experiments.

is among the drugs active against renal cell carcinoma in the extensive list of drugs reviewed by Yagoda et al. ${ }^{12)}$ Therefore we studied the feasibility of the kidney- and site-selective delivery of 5-FU following the application of 5-FU on the kidney surface in rats. Although oral administration of 5-FU has been used for convenience, this administration route has the disadvantage that the oral bioavailability of 5-FU is low and erratic. ${ }^{13,14)}$ Therefore intravenous administration of 5-FU was selected as a control for kidney surface application.

First, we established an experimental system utilizing a cylindrical diffusion cell attached to the right kidney surface in rats (Chart 1). This system enables us to examine drug absorption from the kidney surface without interference by absorption from the other sites. Figure 1 shows the tissue concentration profiles of 5-FU for up to $180 \mathrm{~min}$ after intravenous administration or kidney surface application of 5-FU. After intravenous administration of 5-FU, the concentration of 5-FU in the right and left kidneys was almost the same and the drug was rapidly eliminated (Fig. 1A). After right kidney surface application of 5-FU, on the other hand, the concentration of 5-FU in the right kidney was significantly higher than in the left kidney and other tissues (Fig. 1B), and was specifically detected in the right kidney at 60,120 , and 180 min. Figure 2 shows the intrarenal distribution of 5-FU 120 min after intravenous administration or kidney surface application of 5-FU. To evaluate the intrarenal distribution of 5-FU, the right kidney was divided from the reniportal structure, and were further separated into the site under the diffusion cell and the site not under the diffusion cell (Chart 2). 5FU concentrations in all sites of the right kidney after intravenous administration of 5-FU were almost the same (Fig. 2A). In contrast, the concentration of 5-FU in site 1 after kidney surface application of 5-FU was approximately 4.2-, 2.5, and 6.2-fold higher than those in site 2 , site 3 , and site 4 , respectively (Fig. 2B).

The side effects of 5-FU are important problems to overcome in cancer chemotherapy. The common clinical systemic side effects of 5-FU are myelosuppression, stomatitis, nausea, emesis, and diarrhea after intravenous and/or oral ad- 

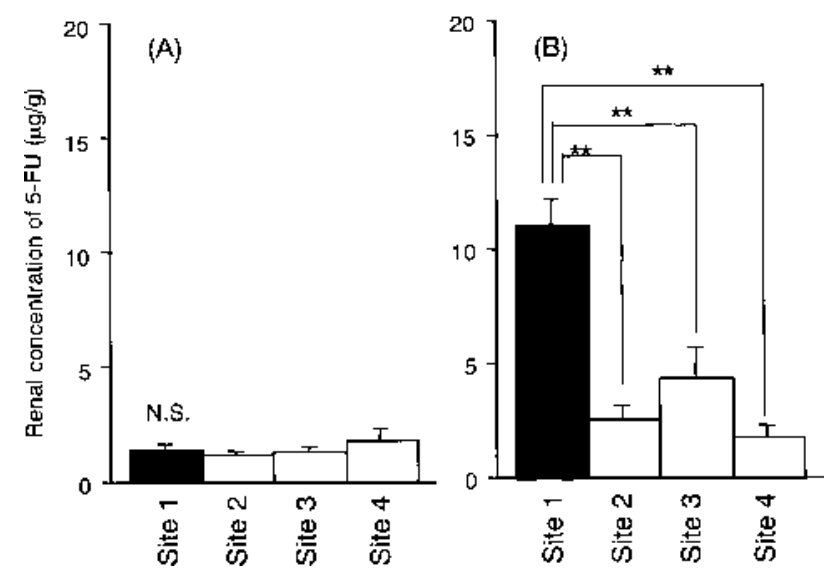

Fig. 2. Intrarenal Concentration of 5-FU 120 Min after Intravenous Administration (A) or Kidney Surface Application (B) of 5-FU to Rats at a Dose of $5 \mathrm{mg}$

Statistical comparisons were performed using analysis of variance $(* * p<0.01$, significantly different from the other sites; N.S., not significant). Each value represents the mean \pm S.E. of at least four experiments.

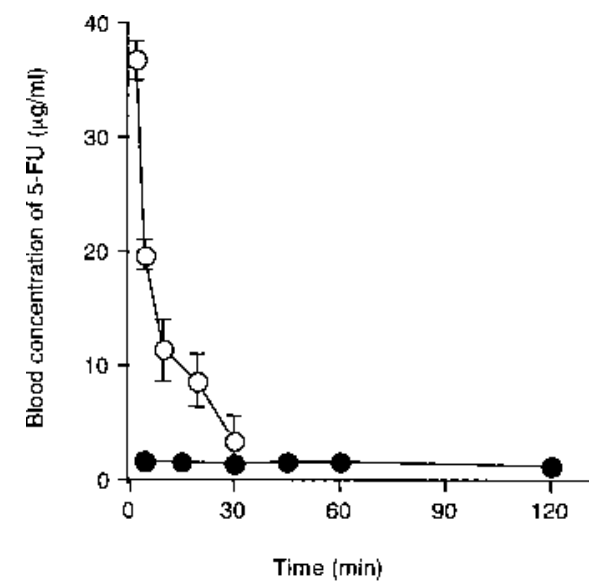

Fig. 3. Blood Concentration of 5-FU after Intravenous Administration $(\bigcirc)$ or Kidney Surface Application ( ) of 5-FU to Rats at a Dose of $5 \mathrm{mg}$

Each value represents the mean \pm S.E. of at least five experiments.

ministration, ${ }^{15)}$ and they might be caused by the high initial blood concentration of 5-FU. Accordingly, the blood concentration profiles of 5-FU after intravenous administration or kidney surface application were evaluated as an index of the systemic side effects of 5-FU (Fig. 3). After kidney surface application of 5-FU, a low blood concentration $(<1.7 \mu \mathrm{g} / \mathrm{ml})$ was seen until $120 \mathrm{~min}$. The maximum blood concentration of 5-FU after kidney surface application was much lower than after intravenous administration. These results suggest that the kidney surface application of 5-FU could diminish the systemic side effects during treatment for renal cell carcinoma.

The direct injection route is not suitable for targeted siteselective drug delivery with high blood flow, because directly injected drugs are rapidly cleared from the injected site, followed by drainage into the systemic circulation. ${ }^{16)}$ On the other hand, 5-FU was site selectively delivered to the kidney utilizing the absorption on the kidney surface (Fig. 1B), because 5-FU was supplied from kidney surface without injuring the kidney. Such information should be useful in the development of a novel administration route for drug delivery to targeted sites in the kidney.

In summary, we demonstrated the kidney- and site-selective delivery of 5-FU utilizing absorption on the kidney surface in rats. Direct injection of drugs can injure the organs with the syringe needle; consequently they were limited to inject the drug continually. In contrast, kidney surface application dose not stress the kidney, and continuous catheter administration may be possible to enable long-term application of 5-FU. Furthermore, kidney- and site-selective drug delivery could be utilized with drugs for the treatment of the various renal diseases as well as renal cell carcinoma.

Acknowledgments This work was supported in part by a Grant-in-Aid for Scientific Research from the Ministry of Education, Culture, Sports, Science and Technology, Japan. This work was also supported in part by a Grant-in-Aid from the Uehara Memorial Foundation. The authors thank Hiromi Shibutani for technical assistance.

\section{REFERENCES}

1) Thompson I. M., Peek M., J. Urol., 140, 487-490 (1988).

2) Motzer R. J., Russo P., J. Urol., 163, $408-417$ (2000).

3) Noda S., Konno S., Tanaka J., Yamada M., Yoshitake N., Anticancer Res., 10, 709-716 (1990).

4) Kobayashi M., Maeda H., Imai K., Konno T., Sugihara S., Yamanaka H., Urology, 37, 288-294 (1991).

5) Kato T., Nemoto R., Mori H., Takahashi M., Tamakawa Y., J. Urol., 125, 19-24 (1981).

6) Kato T., Sato K., Sasaki R., Kakinuma H., Moriyama M., Cancer Chemother. Pharmacol., 37, 289-296 (1996).

7) Kerr D. J., Willmott N., McKillop J. H., Cummings J., Lewi H. J., McArdle C. S., Cancer, 62, 878-883 (1988).

8) McArdle C. S., Lewi H., Hansell D., Kerr D. J., McKillop J., Willmott N., Br. J. Surg., 75, 132-134 (1988).

9) Pyrhönen S., Salminen E., Ruutu M., Lehtonen T., Nurmi M., Tammela T., Juusela H., Rintala E., Hietanen P., Kellokumpu-Lehtinen P.L., J. Clin. Oncol., 17, 2859-2867 (1999).

10) Watanabe J., Hayashi Y., Iwamoto K., Ozeki S., Chem. Pharm. Bull., 33, 1187-1194 (1985).

11) Sawai Y., Yamaoka K., Ito T., Nakagawa T., Biol. Pharm. Bull., 20, 1313-1316 (1997).

12) Yagoda A., Abi-Rached B., Petrylak D., Semin. Oncol., 22, 42-60 (1995).

13) Phillips T. A., Howell A., Grieve R. J., Welling P. G., J. Pharm. Sci., 69, 1428-1431 (1980).

14) Diasio R. B., Harris B. E., Clin. Pharmacokinet., 16, 215-237 (1989).

15) Fata F., Ron I. G., Kemeny N., O’Reilly E., Klimstra D., Kelsen D. P., Cancer, 86, 1129-1134 (1999).

16) Nishida K., Sato N., Sasaki H., Nakamura J., J. Pharm. Pharmacol., 46, 867-870 (1994). 